Trauma Berufskrankh 2016 18 (Suppl 5):S405-S412 DOI 10.1007/s10039-016-0158-1

Online publiziert: 31. Mai 2016

(c) Springer-Verlag Berlin Heidelberg 2016

CrossMark
M. Auerswald · H. Neumann · S. Breer - D. Rundt - S. Fuchs - M. Faschingbauer

BG Klinikum Hamburg, Hamburg, Deutschland

\section{Standards in der Unfallchirurgie} Therapie der proximalen Humerusfraktur
Die proximale Humerusfraktur ist immer noch eine Herausforderung für den Behandelnden. Neben der anspruchsvollen Diagnostik erschweren uneinheitliche Einteilungen und nicht validierte Therapieempfehlungen die Festlegung der jeweiligen Behandlung. Dabei ist zu erwarten, dass die Inzidenz aufgrund des demografischen Wandels eine steigende Tendenz haben wird.

\section{Epidemiologie}

Der Anteil an der Gesamtzahl jährlicher Frakturen beträgt 4-5\% [1]. Betroffen sind überwiegend Patienten in der zweiten Lebenshälfte, wobei die Inzidenz ab dem 40. Lebensjahr [2] eine deutliche Zunahme aufweist. Das Geschlechterverhältnis innerhalb der Patientengruppe änderte sich zwar zwischen 1970 und 2012 von etwa 3,6/1 [3] auf bis $\mathrm{zu}$ 2,3/1 [2], jedoch besteht weiterhin ein Überhang der weiblichen Betroffenen.

\section{Unfallmechanismus}

In der Patientengruppe über 40 Jahre ist der Sturz aus Standhöhe mit etwa 88 \% [3] das häufigste Unfallereignis. Anders als z. B. bei der hüftgelenknahen Femurfraktur handelt es sich bei den betroffenen Patienten überwiegend um aktive, selbstbestimmte Personen, die im häuslichen Tätigkeitsumfeld stürzen. Risikofaktoren dieser Altersgruppe sind Gangunsicherheiten durch orthopädische, neurologische oder internistische Erkrankungen. Ein weiterer Risikofaktor, der häufig die Schwere der Verletzung beeinflusst, ist eine bestehende Osteoporose. Die proximale Humerusfraktur tritt in dieser Altersgruppe eher als Monoverletzung auf.
Im Gegensatz dazu liegt bei jüngeren Patienten in den meisten Fällen ein Hochrasanztrauma zugrunde. Dies tritt überwiegend im Rahmen von Verkehrsunfällen, aber auch beiStürzen aus großer Höhe oder bei Risikosportarten wie Fallschirmspringen auf. In dieser Altersgruppe ist die proximale Humerusfraktur daher eher als Begleitverletzung anzusehen.

\section{Anatomie}

Die ursprünglich von Codman [4] vorgeschlagene Einteilung des proximalen Humerus in 4 Anteile entspricht den 4 Hauptfragmenten, die im Rahmen der Fraktur auftreten können. Dislokationsrichtungen sind auf die ansetzende Muskulatur zurückzuführen. Das Tuberculum-minus-Fragment wird entsprechend durch den Zug des M. subscapularis nach medial gezogen, während das Tuberculum-majus-Fragment nach kraniodorsal disloziert. Durch den Zug des M. pectoralis major kommt es zu einer Dislokation des Humerusschaftes nach medial.

Gerade in letzter Zeit wird die Durchblutung des Humeruskopfes kontrovers diskutiert: Während aus früheren Untersuchungen hervorging, dass die Durchblutung des vorwiegend über die A. circumflexa humeri anterior und deren Endast, die A. arcuata, gewährleistet wird, zeigen neuere Studien, dass der Anteil der A. circumflexa humeri posterior erheblich größer ist, als bisher angenommen. Hettrich et al. [5] konnten mittels Kontrast-MRT (Magnetresonanztomographie) zeigen, dass eine komplette Durchblutung des Humeruskopfes auch bei Ligatur der A. circumflexa humeri anterior fortbesteht und allein über diesen Weg zu bis zu 75,1 \% aufrechterhalten wird.

\section{Klassifikationen}

Nachdem Kocher 1896 [6] erstmals eine Beschreibung unterschiedlicher proximaler Humerusfrakturen veröffentlichte, wurden verschiedene weitere Klassifikationen vorgestellt. So schlug Codman 1934 [4] die Einteilung in 4 Hauptfragmente vor. Darauf aufbauend, zeigte Neer 1970 [7] ein komplexes System, das Einteilungen der Frakturen in „minimal displaced“ bis zu "4-part“ vorsieht. Weiterhin wird der frakturierte Teil beschrieben von „anatomical neck“ über „surgical neck“" und „greater tuberosity“ bis $\mathrm{zu}$ „lesser tuberosity“ und „fracture dislocation“" (• Abb. 1).

Neben der Neer-Klassifikation hat die Arbeitsgemeinschaft für Osteosynthesefragen (AO)-Klassifikation [8] nach Müller die weiteste Verbreitung gefunden. In einem System aus 3 Basistypen („extraartikulär“, „bedingt intraartikulär“, „intraartikulär") werden jeweils 9 Unterteilungen vorgenommen, sodass hier eine detailreiche Einteilung mit 27 Subtypen entstand.

Neueren Datums ist die Klassifikation nach Habermeyer [1] von 1997. Hier werden 5 Frakturtypen $(0, A, B, C, X)$ in 3 Grade unterteilt. Der Typ 0, die nicht verschobene Fraktur, wird als Grad I beschrieben, der Typ X weist 2 Grade auf, wobei der Grad I durch die ventrale Luxation, der Grad II durch die dorsale Luxation charakterisiert ist.

Eine weitere Neuerung stellt die Einteilung nach Hertel [9] von 2005 dar. Die binäre oder sog. „LEGO-Klassifikation“ ist ebenfalls auf die Codman-Klassifikation gegründet, beschreibt jedoch ausschließlich Frakturverläufe, ohne Dislokationen zu berücksichtigen. Hier sind 12 unterschiedliche Frakturformen mög- 


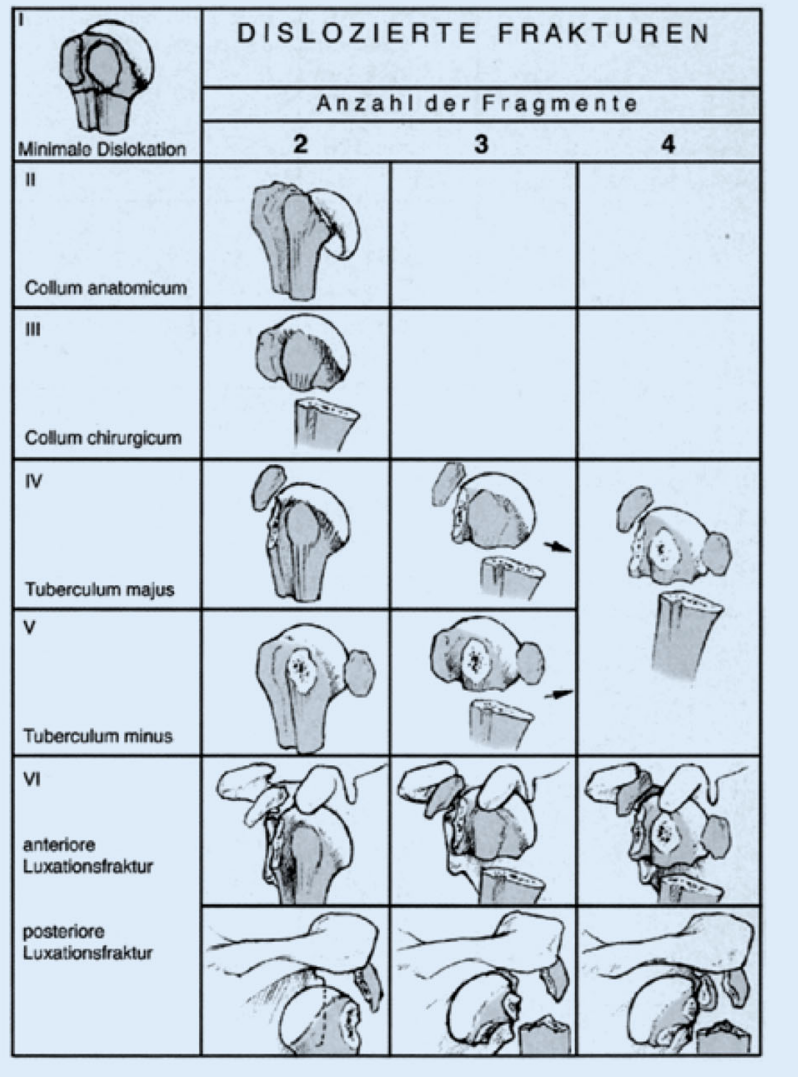

Abb. $1<$ Klassifikation nach Neer [1]

lich. Neben der reinen Frakturklassifikation definiert Hertel Zusatzkriterien [10], die helfen, die zu erwartende Durchblutungssituation des Kalottenfragmentes einzuschätzen:

Zunächst ist die Länge des posteromedialen Metaphysenanteiles von mehr als $8 \mathrm{~mm}$ ein positiver Prädiktor. Der zweite positiv prädiktive Wert ist das Ausmaß der Kalottenfragmentdislokation. Bei einer Dislokation bis zu $2 \mathrm{~mm}$ ist davon auszugehen, dass die mediale Periostbrücke zum Kalottenfragment erhalten geblieben ist.

Mit der kürzlich veröffentlichten Einteilung nach Resch [11] besteht die Möglichkeit, Fehlstellungen in 3 Ebenen $\mathrm{zu}$ klassifizieren: nicht dislozierte Frakturen werden als Typ 1 beschrieben, Typ2-Frakturen zeigen ein anteriores oder posteriores Abkippen, als Typ 3 werden Frakturen in Valgusfehlstellung, als Typ 4 Frakturen in Varusfehlstellung definiert. Der Typ 5 nach Resch bezeichnet die anteriore oder posteriore Dislokation des Humeruskopfes. Weiterhin wird bedarfsweise ein Hauptfragment mithilfe eines zusätzlichen Buchstabens beschrieben (G: Tuberculum majus, L: Tuberculum minus).

Allen bisherigen Klassifikationssystemen wird zum Vorwurf gemacht, dass sie keine gute Inter- und Intraobserverreliabilität aufweisen. So konnten Majed et al. [12] in einem Vergleich der Einteilungen von Neer, AO, Hertel und Resch zeigen, dass in keinem Fall eine Interobserverreliabilität über $\mathrm{k}=0,44$ erreicht werden konnte, was einer nur mittelmäßigen Übereinstimmung [13] entspricht. Diese Ergebnisse konnten hinsichtlich der Neer- und der AO-Klassifikation durch Carrerra et al. [14] 2012 bestätigt werden.

Als Ursache für die fehlende Reproduzierbarkeit wird die diagnostische Grundlage, das konventionelle Röntgen, gesehen. Bei akutverletzten Patienten ist eine wiederholbar achsgerechte Einstellung der betroffenen Extremität selbst unter Analgesie nicht immer möglich. Nicht achsgerechte Aufnahmen haben jedoch nicht den benötigten Aussagewert. Selbst durch die konventionelle 2-D-Computertomographie konnte kei- ne entscheidende Verbesserung der Reproduzierbarkeit erreicht werden [15]. Erst durch die Einführung von 3-D-Rekonstruktionen konnten hier signifikante Verbesserungen erzielt werden. Weitere Erfolge wurden durch die Nutzung stereovisualisierter 3-D-Volume-Rendering-Datensätze erzielt [15], wobei diese Technik bisher noch keine klinisch relevante Verbreitung gefunden hat.

\section{Therapie}

Die therapeutischen Ziele sind neben dem Erhalt oder der Verbesserung der Kopfdurchblutung die komplikationslose Heilung in möglichst anatomischer Stellung sowie das Erhalten oder Erzielen einer primären Übungsstabilität. Jedoch bestehen weiterhin keine evidenzbasierten Behandlungsrichtlinien.

Aus einem kürzlich erschienenen Cochrane Review [16] geht hervor, dass auch in unserer Zeit keine Nachweise für die Überlegenheit des konservativen Vorgehens gegenüber der operativen Behandlung bestehen, ebenso fehlten bisher Hinweise für die Überlegenheit eines speziellen operativen Verfahrens [17]. Als ursächlich hierfür wird einvernehmlich die Vielzahl an Studiendesign, Versorgungsmöglichkeiten und Evaluationshilfsmitteln gesehen, sodass eine Vergleichbarkeit im größerem Umfang praktisch nicht zu erreichen ist [18].

Verschiedene Veröffentlichungen heben jeweils einzelne therapeutische Aspekte hervor. So konnten Misra et al. [19] 2001 im Rahmen einer Übersichtsarbeit zeigen, dass hinsichtlich Schmerzlinderung und Wiedererlangen des Bewegungsausmaßes die konservative Behandlung der operativen unterlegen ist, jedoch mit einer geringeren Infektionsrate einhergeht. Demgegenüber publizierten Hanson et al. [20] 2009 gute Ergebnisse der konservativen Behandlung von 160 Patienten mit Verletzungen von überwiegend geringerem Schweregrad bezüglich Heilung und funktionellem Ergebnis. Es konnte im Constant Score ein Unterschied von im Mittel 8,2 Punkten beim Vergleich der verletzten mit der unverletzten Schulter gefunden werden. Fjalestad et al. [21] konnten bei B2- und C2-Frakturen keine 
Unterschiede der operativen Behandlung mittels winkelstabiler Plattenosteosynthese im Vergleich zur konservativen Behandlung bezüglich des funktionellen Ergebnisses darstellen.

Bei der Wahl der passenden Therapieform sind Begleitfaktoren zu berücksichtigen. So spielen neben dem biologischen Alter Nebenerkrankungen und deren ggf. bestehende medikamentöse Therapie und mögliche, im Rahmen des zugrunde liegenden Unfalles aufgetretene Begleitverletzungen eine Rolle. Gerade wegen der fehlenden Therapierichtlinien gewinnt die Berücksichtigung dieser Faktoren an Bedeutung. Klassische Beispiele sind z. B. die medikamentöse Therapie zur Antikoagulation oder die Therapie mit Biguaniden. Weiterhin sind die Weichteilverhältnisse zu berücksichtigen, der Anspruch, aber auch die zu erwartende Mitarbeit der Patienten spielen eine Rolle in der Entscheidungsfindung.

Im klinischen Alltag hat sich als grober Anhalt ein Vorgehen etabliert, das an die Neer-Klassifikation angelehnt ist. So werden Neer-I-Frakturen rein konservativ behandelt. Neer-II-Frakturen werden unter Berücksichtigung der oben genannten Begleitfaktoren und Analyse der Fraktur operativ oder konservativ behandelt, während Neer-III- und -IV-Frakturen die klare Domäne der operativen Behandlung sind.

Bei der Beurteilung der Neer-II-Frakturen sind die Grenzwerte zur Dislokation besonders zu berücksichtigen. Neer [7] sah als Grenze zwischen konservativer und operativer Behandlung eine Dislokation von $>1 \mathrm{~cm}$ sowie eine Kopfabkippung von $>45^{\circ}$ an. Jedoch sind diese Werte im Hinblick auf den demografischen Wandel sicherlich differenziert $\mathrm{zu}$ sehen. Daher sind bei biologisch jüngeren Patienten die von Lill [22] definierten Grenzwerte zu berücksichtigen. Hier wird die Grenze zum operativen Vorgehen bereits bei $0,5 \mathrm{~cm}$ Dislokation, $20^{\circ}$ Kopfabkippung und zusätzlich $0,2 \mathrm{~cm} \mathrm{Tu}$ berkulumdislokation gesehen.

Eindeutige Indikationen für die operative Therapie sind die metaphysäre Trümmerzone, jede Form der Luxations- und alle offenen Frakturen. Ebenso sind die Fraktur des Collum anato-

Trauma Berufskrankh 2016 · 18 (Suppl 5):S405-S412 DOI 10.1007/s10039-016-0158-1

(c) Springer-Verlag Berlin Heidelberg 2016

\section{Auerswald $\cdot$ H. Neumann $\cdot$ S. Breer $\cdot$ D. Rundt $\cdot$ S. Fuchs $\cdot$ M. Faschingbauer}

\section{Standards in der Unfallchirurgie. Therapie der proximalen Humerusfraktur}

\section{Zusammenfassung}

Die proximale Humerusfraktur betrifft zum einen junge Menschen, die diese Verletzung im Rahmen von Hochrasanztraumata erleiden, mehrheitlich aber Patienten in der zweiten Lebenshälfte, bei denen sie nach der hüftgelenknahen Femurfraktur und der distalen Radiusfraktur zu den häufigsten Frakturen zählt. Die Mehrzahl der Betroffenen in dieser Altersgruppe ist weiblich, jedoch zeigt sich eine Zunahme in der männlichen Bevölkerung. Die bestehenden Klassifikationssysteme weisen eine mangelhafte Reproduzierbarkeit auf. Derzeit besteht weder Konsens hinsichtlich der Überlegenheit der operativen über die konservative Therapieform, noch hinsichtlich der Überlegenheit eines bestimmten operativen Versorgungsverfahrens. Die größten
Änderungen im Hinblick auf die operative Versorgung werden aktuell auf dem Gebiet der endoprothetischen Versorgung gesehen. Es zeichnet sich ein zunehmender Trend ab weg von der anatomischen hin zur inversen Prothese. Auch wird die Indikation zur inversen Prothese zunehmend bei jüngeren, noch berufstätigen Menschen gestellt. Die vorliegende Darstellung soll einen Überblick über die derzeitigen Indikationen und Therapiemöglichkeiten mit Schwerpunkt auf der operativen Therapie der mehrfragmentären proximalen Humerusfraktur geben.

Schlüsselwörter

Klassifikation · Implantate · Winkelstabilität . Endoprothese - Operation

\section{Standards in trauma surgery. Treatment of proximal humeral fractures}

\section{Abstract}

Proximal humeral fractures (PHF) also affect younger people who suffer such injuries due to high-velocity trauma but mainly involve people in the second half of life. In this age group PHF are the most frequent fractures following distal radius fractures. The majority of those affected in this age group are female but an increase in the male population has been observed. The existing classification systems show a lack of reproducibility and currently there is a lack of consensus with respect to the superiority of surgical treatment compared to conservative therapy as well as the superiority of one particular operative procedure. The greatest changes with respect to operative procedures can currently be observed in the field of prosthetic treatment. There is an increasing trend away from anatomical and towards inverse prostheses. The indications for inverse prostheses are increasingly directed towards younger people who are still in employment. This article gives a survey over current indications and therapeutic concepts for PHF with a focus on the operative treatment of comminuted fractures.

\section{Keywords}

Classification · Surgery · Implants · Angle stability Prosthesis micum und die „head-split-fracture“ Indikationen für die operative Therapie.

\section{Konservative Behandlung}

Bis $\mathrm{zu} 85 \%$ der proximalen Humerusfrakturen sind nicht oder nur gering disloziert $[1,7]$ und können konservativ behandelt werden, ohne langfristig schlechte Ergebnisse aufzuweisen. Hanson et al. [20] beschreiben in einem Kollektiv aus überwiegend Neer-I- und -II-Frakturen eine Quote von 97,6\% Patienten, die nach konservativer Behandlung in die Berufstätigkeit zurückkehrten. Vorteilhaft ist die Möglichkeit, die Behandlung auf ambulanter Basis durchzuführen. Die Gefahr von perioperativen Komplikationen wie Gefäß- und Nervenläsionen, Infektion und Wundheilungsstörungen ist minimiert, zum Teil sogar ausgeschlossen. Demgegenüber bestehen die Nachteile der eingeschränkten Repositionsmöglichkeit, prolongierter Schmerzhaf- 

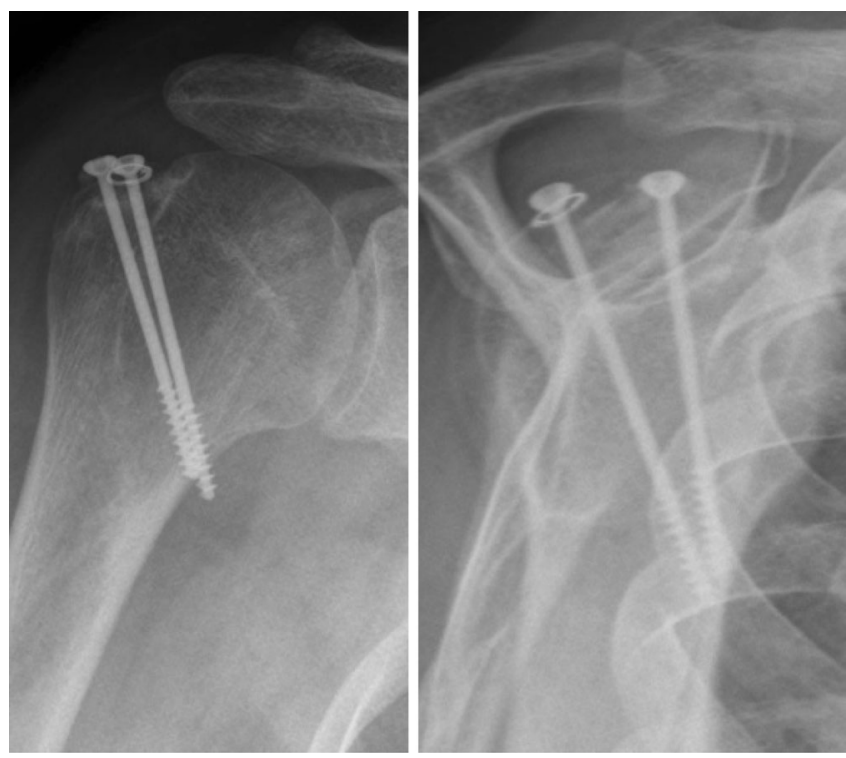

Abb. $2 \Delta$ Zugschraubenosteosynthese einer isolierten Tuberculum-majus-Fraktur

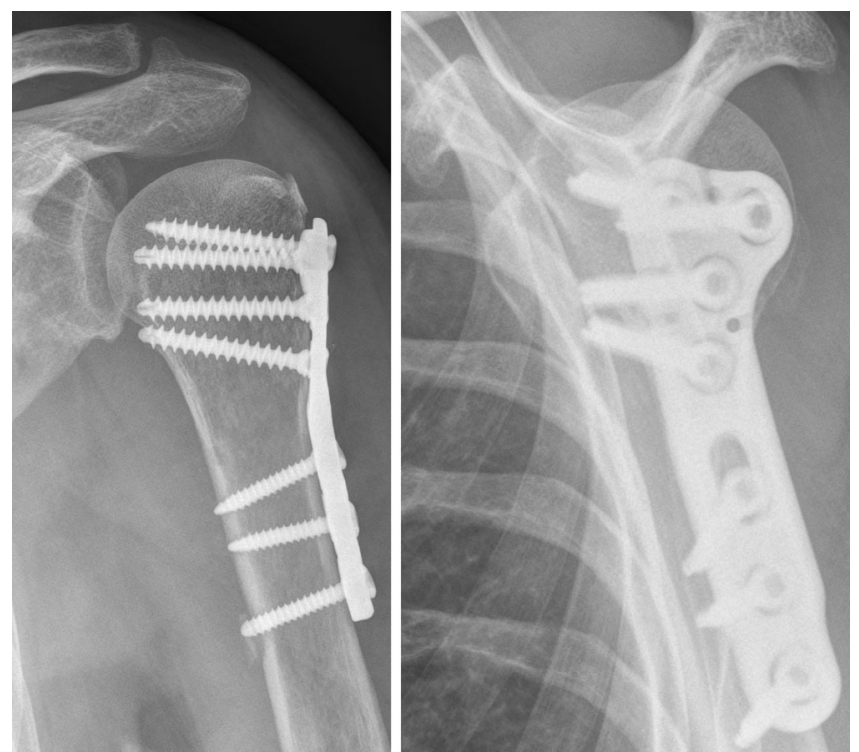

Abb. $3 \Delta$ Multidirektional winkelstabile Plattenosteosynthese tigkeit mit der Gefahr der Ausbildung eines chronisch regionalen Schmerzsyndromes (CRPS). Hier ist eine Abwägung zu treffen, um dem Betroffenen die angemessene Behandlung anbieten zu können.

\section{Operative Behandlung}

Die Indikation zur operativen Intervention besteht bei Dislokationen oberhalb der beschriebenen Grenzwerte. Außerdem gelten als absolute Operationsindikation die metaphysäre Trümmerzone, offene und Luxationsfrakturen, Frakturen des Collum anatomicum und "headsplit-fractures“ sowie Gefäß- oder Nervenläsionen.

Die Möglichkeiten der operativen Versorgung sind umfangreich. Grundsätzlich ist zwischen kopferhaltenden und -resezierenden Verfahren zu unterscheiden. Zur Wahl steht von arthroskopisch assistierten Verfahren mit einzelnen Schrauben bis hin zur primären Implantation einer inversen Prothese eine große Anzahl an Implantaten.

\section{Zugang}

Die minimalinvasivste Methode zur Reposition von Frakturen des proximalen Humerus ist sicher das arthroskopische Vorgehen. Der Patient ist in Beach-chairLagerung positioniert. Da ein Umstieg auf das offene Vorgehen nicht auszuschließen ist, sollte auf eine Seitenlagerung zur Arthroskopie verzichtet werden. Als Standard werden das posteriore sowie das anterosuperiore, bedarfsweise auch das laterale Portal genutzt.

Die Standardzugänge für die offene Reposition sind zum einen der deltoideopektorale Zugang nach Kocher, zum anderen der anterolaterale oder Delta-split-Zugang. Beide Zugänge weisen Vor- und Nachteile auf. So kann über einen deltoideopektoralen Zugang eine gute Übersicht über die Kalotte und das Tuberculum minus gewonnen werde. Er bietet ausreichenden Platz zur Armierung der Tuberkula, was die Fixierung beideram Osteosynthesematerial ermöglicht. Weiterhin ist - im Hinblick auf die Versorgung höhergradiger Frakturformen - der Umstieg auf eine prothetische Versorgung gut möglich. Da zwischen $M$. deltoideus und $M$. pectoralis major eingegangen wird, kommt es zu keiner nennenswerten Weichteiltraumatisierung. Allenfalls die Darstellbarkeit der Tuberculum-majus-Region kann über diesen Zugang eingeschränkt sein.

Der Delta-Split ist gerade für Pathologien der Tuberculum-majus-Region sehr geeignet. Das Weichteiltrauma ist ebenso gering wie beim deltoideopektoralen Zugang, da der Muskel in Faserrichtung gespalten wird. Jedoch bringt auch dieser
Zugang Nachteile mit sich: So ist hier die Einstellung des Kalottenfragmentes nicht möglich. Die Tuberculum-minus-Region ist ebenfalls nicht ausreichend darstellbar. Der N. axillaris ist verletzungsgefährdet, und der Umstieg auf eine prothetische Versorgung ist schwer möglich.

\section{Zugschraubenosteosynthese}

Isolierte Frakturen des Tuberculum majus machen etwa $17-21 \%$ aller proximalen Humerusfrakturen aus [23]. Abhängig von der Frakturanalyse ist diese Frakturform ideal arthroskopisch assistiert zu versorgen. Neben der Stabilisierung mit Zugschrauben (• Abb. 2) ist hier auch die Stabilisierung durch Fadenanker wie bei der arthroskopischen Rotatorenmanschettenrefixation möglich.

Als seltene Indikation sind individuell anzupassende Implantate auf dem Markt, die zur Stabilisierung von Trümmerzonen der Tuberculum-majusRegion einsetzbar sind. Dieses Implantat ermöglicht eine gute Stabilisierung dieser Region und bietet zudem die Möglichkeit, das Tuberculum-majus-Fragment $\mathrm{zu}$ sichern. Zur Plattenosteosynthese dieser Frakturform veröffentlichten Gillespie et al. [24] 2015 Ergebnisse mit einem mittleren Follow-up von $27 \mathrm{Mo}-$ naten, die ermutigend sind. 


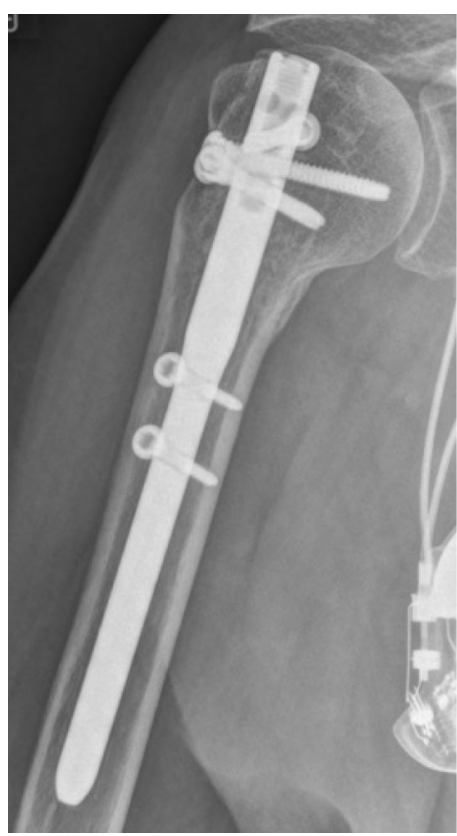

Abb. 4 \ Proximaler Humerusmarknagel

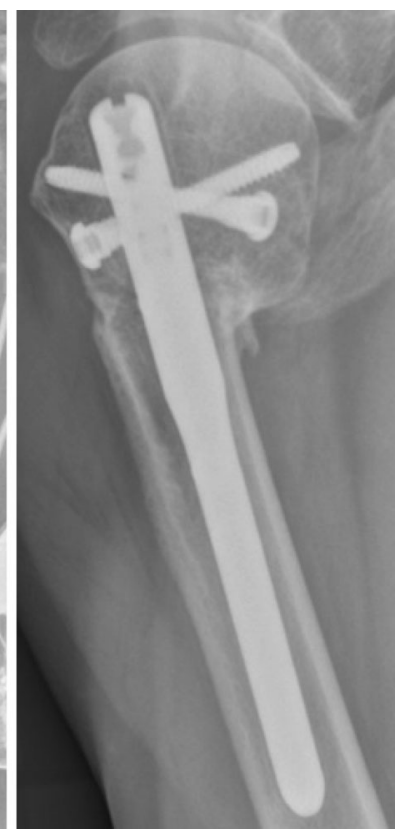

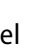
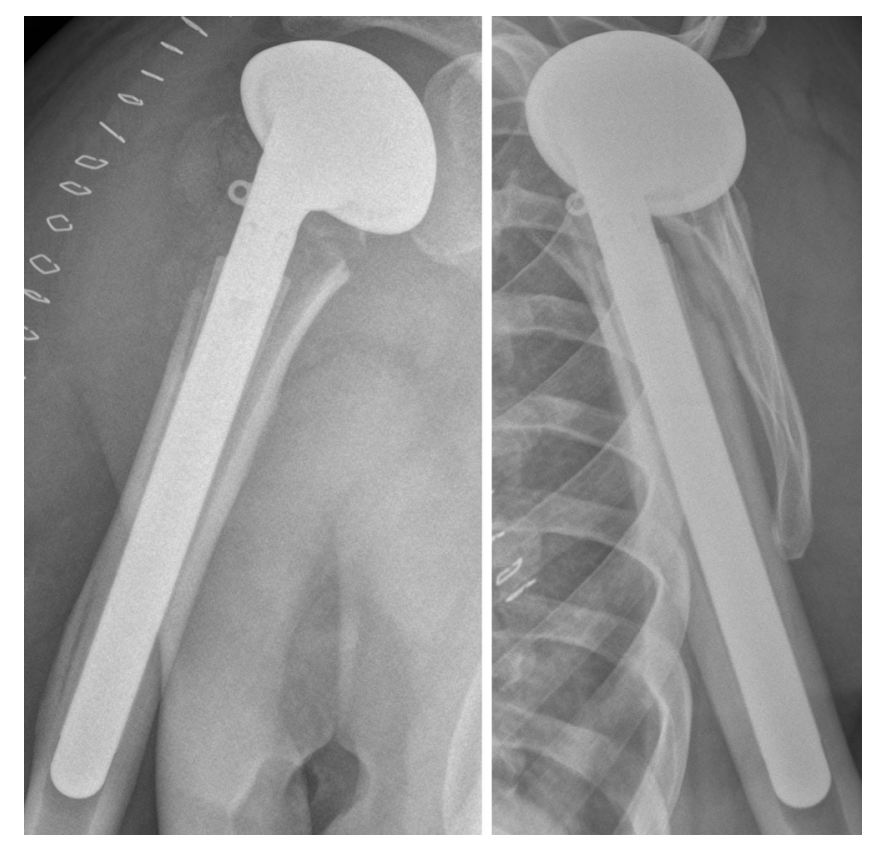

Abb. $5 \Delta$ Anatomische Hemiprothese

\section{Plattenosteosynthese}

Die winkelstabile Plattenosteosynthese hat die konventionellen Platten bei der Versorgung proximaler Humerusfrakturen verdrängt [25] und ist derzeit als das gängigste Implantat anzusehen. Das Prinzip der Winkelstabilität weist eine höhere Stabilität gerade im osteoporotischen Knochen auf [20, 26] und lässt sich offen wie minimalinvasiv umsetzen [27]. Es gibt jedoch Hinweise auf bessere Ergebnisse beim offenen Vorgehen [28]. Eine anatomische Reposition lässt sich häufig minimalinvasiv nicht erzielen. Vor allem bei jungen Patienten ist dies das Implantat der Wahl [29]. Durch die Einführung multidirektional winkelstabiler Platten konnte eine größtmögliche Variabilität bei der Platzierung, v. a. der Kopfschrauben, erreicht werden ([30]; - Abb. 3). Damit ist es möglich, auch Mehrfragmentfrakturen stabil zu versorgen. Jedoch bringt diese erhöhte Stabilität den Nachteil des Cut-out der Schrauben aus dem Humeruskopf mit sich. Zusammenfassend lässt sich jedoch konstatieren, dass eine anatomische Reposition auch bei Mehrfragmentfrakturen nur im Rahmen einer plattenosteosynthetischen Versorgung sicher möglich ist.

\section{Marknagel}

Als weitverbreitetes Implantat sind unterschiedliche Ausführungen intramedullärer Kraftträger auf dem Markt. Neben der winkelstabilen Platte ist der Marknagel (• Abb.4) das am häufigsten genutzte Implantat. Vorteilhaft ist sicherlich die minimalinvasive Vorgehensweise bei der Implantation. Jedoch besteht durch den obligaten Zugang durch den M. supraspinatus oder dessen Sehne eine nicht unerhebliche Zugangsmorbidität [31, 32]. Nachteilig erscheint weiterhin die eingeschränkte Möglichkeit der Reposition und Stabilisierung der Tuberkula [33]. Beider Versorgung von Neer-II- und -IIIFrakturen sind hinsichtlich des funktionellen Ergebnisses bei diesen Frakturformen gute und befriedigende Ergebnisse veröffentlicht worden [32]. Jedoch wird in der gleichen Untersuchung über schlechte Ergebnisse bei Neer IV-Frakturen berichtet mit einem mittleren Constant Score von 23 im Vergleich zur Altersklasse.

\section{Prothese}

Die Indikation zur primären prothetischen Versorgung wird zunehmend weiter gestellt. Es muss jedoch zwischen anatomischer (• Abb. 5) und inverser Prothese (• Abb. 6) differenziert werden.
Waren anatomische Prothesenmodelle über lange Zeit die Standardprothese bei der primären Frakturversorgung, so werden sie zunehmend kritisch hinsichtlich ihres funktionellen Ergebnisses gesehen. Die primäre Versorgung mit einer inversen Prothese war lange betagten Patienten mit geringem Anspruch an das funktionelle Ergebnis vorbehalten. Jedoch werden gerade in neuerer Zeit aufgrund ermutigender Ergebnisse zunehmend inverse Versorgungen auch bei jüngeren, z. T. berufstätigen Patienten vorgenommen [34]. So empfiehlt Lill (persönliche Mitteilungen 2016) die primäre Implantation einer inversen Prothese durchaus auch bei 50 -jährigen Patienten. Wang et al. [35] konnten in einer Metaanalyse zeigen, dass inverse Prothesendesigns den anatomischen hinsichtlich Komplikationshäufigkeit, Einheilungsrate der Tuberkula und Bewegungsausmaß überlegen sind. Hier ist allerdings kritisch anzumerken, dass mit der Implantation einer inversen Prothese bereits die höchste Stufe der prothetischen Versorgung erreicht ist. Eine weitere Eskalation ist nicht möglich, bei Versagen bleibt nur die GirdlestoneSituation. 

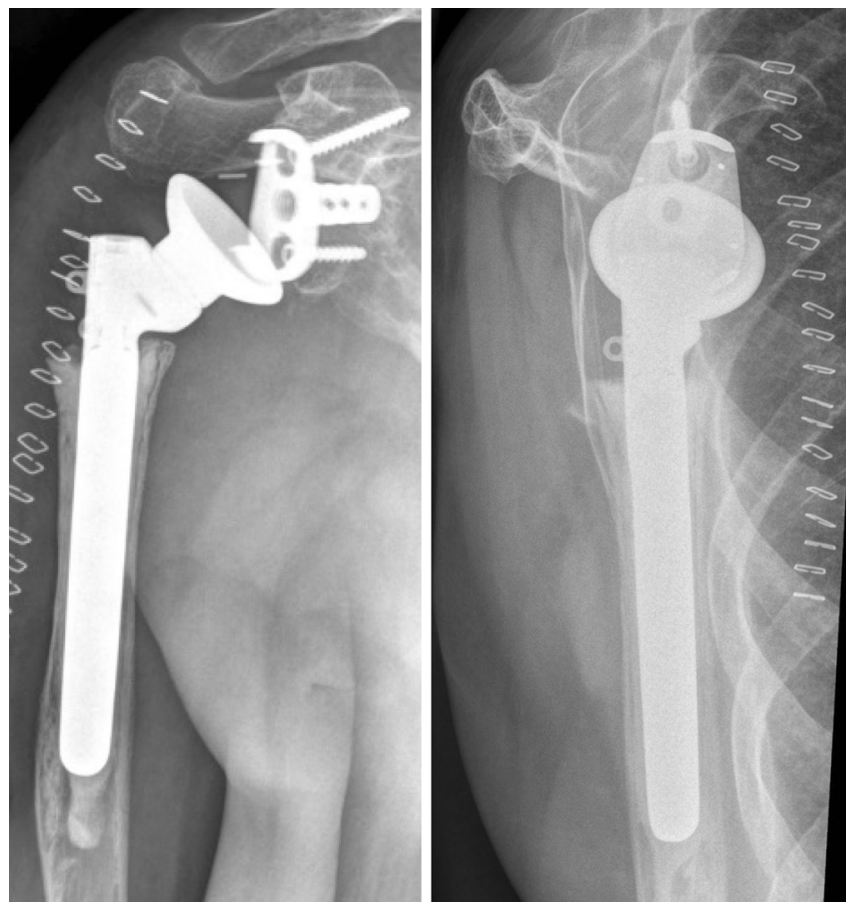

Abb. $6<$ Inverse Prothese

\section{Komplikationen}

Alle Formen der Behandlung proximaler Humerusfrakturen bergen die Gefahr von Komplikationen. Während die überwiegende Anzahl unspezifisch ist, treten einige nur bei speziellen Verfahren auf. Daher ist es sinnvoll, die unerwünschten Verläufe in Bezug auf das gewählte Verfahren zu betrachten.

Die konservative Behandlung wird in der überwiegenden Zahl aller proximaler Humerusfrakturen durchgeführt. Auch hier bestehen relevante Komplikationsmöglichkeiten. So beschreiben Hanson et al. [20] 10,6 \% Dislokationen bzw. Stellungsverluste nach geschlossener Reposition, weiterhin wird von 6,9\% Impingement und Schultersteife berichtet. Darüber hinaus trat eine Pseudarthrosenrate von $7 \%$ auf. Eine Metaanalyse aus dem Jahr 2015 [36] zeigte zusätzlich eine insgesamt signifikant höhere Schmerzhaftigkeit der konservativ behandelten Patienten im Vergleich zu den operierten. Infektionen, avaskuläre Humeruskopfnekrosen, Pseudarthrosen und posttraumatischer Omarthrose traten in vergleichbarer Häufigkeit bei operierten wie bei konservativ behandelten Patienten auf.

Auf der Seite der operativen Verfahren ist zwischen Komplikationen nach kopferhaltenden Verfahren und prothetischer Versorgung zu differenzieren.

In der erste Gruppe sind Komplikationsraten von bis zu $30 \%$ beschrieben $[27,37,38]$. Regelmäßig treten die nichtanatomische Reposition, Materialfehllage, avaskuläre Humeruskopfnekrose, Infektion und Pseudarthrosenbildung [37] auf. Weiterhin bestehen Gefahren wie sekundäre Fragmentdislokation [38], Gelenksteife und posttraumatische Omarthrose.

Zusätzlich werden bei einzelnen Implantaten spezielle Komplikationen dargestellt.

Bei der Versorgung mit Marknägeln ist das subakromiale Impingement eine häufig genannte Komplikation, während der Cut-out der Schrauben aus dem Kalottenfragment häufig bei der winkelstabilen Plattenosteosynthese genannt wird [37, 38].

Die prothetische Versorgung weist als gravierende Komplikation die Resorption der Tuberkula auf. Daneben besteht die Möglichkeit der Prothesenfehlrotation und der inadäquaten Dimensionierung insbesondere der Hemisphäre.

\section{Schlussfolgerungen und Empfehlungen}

Die Versorgung proximaler Humerusfrakturen bleibt eine Herausforderung.
Einigkeit besteht darin, nicht dislozierte Frakturen konservativ zu behandeln. Dies trifft zwar für den Großteil zu, sobald jedoch eine Dislokation außerhalb der von Neer bzw. von Lill definierten Grenzen auftritt, besteht Uneinigkeit hinsichtlich des operativen Verfahrens.

Im klinischen Alltag haben sich verschiedene Behandlungsstränge etabliert. So ist die Fraktur jüngerer Patienten nach anatomischer Reposition plattenosteosynthetisch zu versorgen, während mehrfragmentäre Frakturen älterer Patienten inzwischen als klare Indikation zur primären Implantation einer inversen Prothese gelten. Dislozierte NeerII-Frakturen sind der Marknagelosteosynthese gut zugänglich, können jedoch ebenso sicher plattenosteosynthetisch versorgt werden. Isolierte Tuberculummajus-Abrisse können minimalinvasiv durch Zugschrauben stabilisiert werden, eine vergleichbare Stabilisierung gelingt jedoch ebenso durch arthroskopisch assistiert eingebrachte Fadenanker. Ein Großteil der mehrfragmentären NeerIV-Frakturen kann plattenosteosynthetisch gut versorgt werden, bei älteren Patienten ist aufgrund der eingeschränkten Knochenqualität die Komplikationsrate und damit das funktionelle Endergebnis dem der primären Schulterendoprothese unterlegen. Die Hemiarthroplastie wiederum zeigt im Vergleich zur primären inversen Prothese schlechtere Ergebnisse hinsichtlich des Langzeitergebnisses. Die Herausforderung liegt also nicht allein in der häufig komplexen Versorgung, sondern auch in der Entscheidung, welche Behandlung vorgenommen werden soll. Eine Cochrane-Analyse kam 2010 zu dem Schluss, dass aufgrund der bestehenden Studienlage keine Empfehlungen gegeben werden könnten, es fehle weiterhin an randomisierten Studien [17].

Ein Anhalt, wie bei dislozierten Frakturen vorgegangen werden soll, kann anhand von Zielen definiert werden. So ist das Ziel der operativen Intervention die übungsstabile Osteosynthese. Weitere Voraussetzungen für eine erfolgreiche Behandlung sind die anatomische Reposition und die gewebe/durchblutungschonende Präparation $[18,31]$. 
Beiden Prämissen sind sicherlich mit der offenen Reposition und Stabilisierung mittels winkelstabiler Plattenosteosynthese ideal umgesetzt. Die Marknagelosteosynthese hingegen stößt bei mehrfragmentären Frakturen häufig an ihre technischen Grenzen. Bei dislozierten Neer-II-Frakturen stellt der Marknagel jedoch ein angemessenes Implantat dar.

Die prothetische Versorgung vielfragmentärer Frakturen ist schon seit Langem etabliert. Wurde als primäres Implantat bislang jedoch die anatomische Prothese favorisiert, wird zunehmend die inverse Prothese als Implantat der Wahl gesehen.

Die Hemiarthroplastie bedarf zwingend der intakten Rotatorenmanschette und durchbluteten Tuberkula, die sicher an der Prothese befestigt werden müssen, um eine Einheilung an der gewünschten Stelle zu ermöglichen. Kommt es jedoch zur Resorption der Tuberkula und damit zur Funktionslosigkeit der Rotatorenmanschette, ist die Indikation zum Umstieg auf eine inverse Prothesenvariante gegeben. Diese Problematik führte zur primären Implantation inverser Prothesen. Eine aktuelle Metaanalyse zeigt mit einem Follow-up von bis zu 43,2 Monaten auch international deutlich bessere Ergebnisse nach Implantation inverser Prothesen verglichen mit anatomischen Modellen [35].

Hier zeichnet sich möglicherweise ein Trend $a b$, der eine Verbesserung in der Versorgung proximaler Humerusfrakturen ausmacht. Es bleibt jedoch weiterhin die Notwendigkeit bestehen, klare Indikationen für die unterschiedlichen osteosynthetischen Versorgungsarten durch randomisierte Studien deutlich herauszuarbeiten.

\section{Fazit für die Praxis}

- Nicht dislozierte proximale Humerusfrakturen sollten konservativ behandelt werden.

- Ab einer Dislokation von 0,5 cm, $20^{\circ}$ Kopfabkippung sowie $0,2 \mathrm{~cm}$ Tuberkulumdislokation besteht eine Operationsindikation.

- Ziel der operativen Versorgung ist die primäre Übungsstabilität bei anatomischer Reposition. Beide Forderungen werden am sichersten durch eine offene Reposition und winkelstabile Fixation erfüllt.

- Die konventionelle Plattenosteosynthese hat keinen Stellenwert in der Versorgung der proximalen $\mathrm{Hu}$ merusfraktur und sollte nicht mehr durchgeführt werden.

- Im Hinblick auf prothetische Versorgungen besteht ein Trend zur primären Implantation inverser Prothesen, kritisch ist hier zu bemerken, dass bei Versagen dieser Prothesenform die rekonstruktiven Therapieformen ausgereizt sind.

- Insgesamt besteht der dringende Bedarf an prospektiv randomisierten Untersuchungen, um eindeutige Behandlungsformen festlegen zu können.

\section{Korrespondenzadresse}

\section{Auerswald}

BG Klinikum Hamburg

Bergedorfer Str. 10, 21033 Hamburg,

Deutschland

m.auerswald@bgk-hamburg.de

\section{Einhaltung ethischer Richtlinien}

Interessenkonflikt. M. Auerswald, H. Neumann, S. Breer, D. Rundt, S. Fuchs und M. Faschingbauer geben an, dass kein Interessenkonflikt besteht.

Dieser Beitrag beinhaltet keine von den Autoren durchgeführten Studien an Menschen oder Tieren.

The supplement containing this article is not sponsored by industry.

\section{Literatur}

1. Habermeyer P (1997) Die Humeruskopffraktur. Unfallchirurg 100:820-837

2. Kim SH, Szabo RM, Marder RA (2008) Epidemiology of humerus fractures in the United States: nationwide emergency department sample. Arthritis Care Res 64(2012):407-414

3. Palvanen M, Kannus P, Niemi S, Parkkari J (2006) Update in the epidemiology of proximal humeral fractures. Clin Orthop Relat Res 442:87-92

4. Codman EA (1934) The Shoulder: Rupture of the supraspinatus tendon and other lesions in or about the subacromial bursa. T. Todd Company, Boston

5. Hettrich CMetal (2010) Quantitative assessment of the vascularity of the proximal part of the humerus. JBone Joint Surg Am 92:943-948

6. Kocher T (1896) Beiträge zur Kenntnis einiger Praktisch wichtiger Fracturformen. Carl Sallmann, Basel Leipzig
7. Neer CS (1970) Displaced proximal humeral fractures. I. Classification and evaluation. J Bone Joint Surg Am 52:1077-1089

8. Müller ME, Nazarian S, Koch P, Schatzker J (1990) The comprehensive classification of fractures of long bones. Springer-Verlag, Berlin Heidelberg

9. Hertel R (2005) Fractures of the proximal humerus in osteoporotic bone. Osteoporos Int 16(Suppl 2):S65-S72

10. Hertel R, Hempfing A, Stiehler M, Leunig M (2004) Predictors of humeral head ischemia after intracapsular fracture of the proximal humerus. JShoulder Elbow Surg 13:427-433

11. Resch $\mathrm{H}$ et al (2015) Classification of proximal humeral fractures based on a pathomorphologic analysis. J Shoulder Elbow Surg. doi:10.1016/j.jse.2015.08.006

12. Majed A et al (2011) Proximal humeral fracture classification systems revisited. J Shoulder Elbow Surg 20:1125-1132

13. Landis JR, Koch GG (1977) An application of hierarchical kappa-type statistics in the assessment of majority agreement among multiple observers. Biometrics 33:363-374

14. Carrerra EF da, Wajnsztejn A, Lenza M, Netto NA (2012) Reproducibility of three classifications of proximal humeral fractures. Einstein (Sao Paulo) 10:473-479

15. Brunner A, Honigmann P, Treumann T, Babst $R$ (2009) The impact of stereo-visualisation of three-dimensional CT datasets on the inter- and intraobserver reliability of the AO/OTA and Neer classifications in the assessment of fractures of the proximal humerus. JBone Joint Surg Br 91:766-77

16. Handoll HHG, Brorson S (2015) Interventions for treating proximal humeral fractures in adults. Cochrane Database Syst Rev 11:CD000434

17. Rangan A et al (2015) Surgical vs nonsurgical treatment of adults with displaced fractures of the proximal humerus: the PROFHER randomized clinical trial. JAMA 313:1037-1047

18. Burkhart KJ, Dietz SO, Bastian L, Thelen U, Hoffmann R, Müller LP (2013) Behandlung der proximalen Humerusfraktur des Erwachsenen. Dtsch Arztebl Int 110:591-597

19. Misra A, Kapur R, Maffulli N (2001) Complex proximal humeral fractures in adults-a systematic review of management. Injury 32:363-372

20. Hanson B, Neidenbach P, Boer P de, Stengel D (2009) Functional outcomes after nonoperative management offractures of the proximal humerus. JShoulderElbow Surg 18:612-621

21. Fjalestad T, Hole $M \emptyset$, Hovden $I A H$, Blücher J, Strømsøe K (2012) Surgical treatment with an angular stable plate for complex displaced proximal humeral fractures in elderly patients: a randomized controlled trial. J Orthop Trauma 26:98-106

22. Lill H, Voigt C (2010) Proximale Humerusfraktur. Z Orthop Unfall 148:353-360 (quiz 361-2)

23. Bahrs C, Lingenfelter $E$, Fischer F, Walters EM, Schnabel M (2006) Mechanism of injury and morphology of the greater tuberosity fracture. JShoulderElbow Surg 15:140-147

24. Gillespie RJ, Johnston PS, Gordon VA, Ward PJ, Getz CL (2015) Using plate osteosynthesis to treat isolated greater tuberosity fractures. Am J Orthop 44:E248-51

25. Ring D (2007) Current concepts in plate and screw fixation of osteoporotic proximal humerus fractures. Injury 38(Suppl 3):S59-S68

26. Wolter D, Schümann U, Seide K (1999) Unisverseller Titanfixateur intern. Trauma Berufskrankh 1:307-319 
27. Falez F et al (2016) Minimally invasive plate osteosynthesis in proximal humeral fractures: oneyear results of a prospective multicenter study. Int Orthop 40:579-585

28. Sohn H-S, Shin S-J (2014) Minimally invasive plate osteosynthesis for proximal humeral fractures: clinical and radiologic outcomes according to fracture type. JShoulder Elbow Surg 23:1334-1340

29. Sproul RC, lyengar JJ, Devcic Z, Feeley BT (2011) A systematic review of locking plate fixation of proximal humerus fractures. Injury 42:408-413

30. Wurm M, Wolter D, Wenzl M, Jessel M (1999) Erste klinische Erfahrungen mit einem Fixateur interne am Humerus. Trauma Berufskrankh 1:370-374

31. DilisioMF, NowinskiRJ, Hatzidakis AM, FehringerEV (2016) Intramedullary nailing of the proximal humerus: evolution, technique, and results. J ShoulderElbow Surg. doi:10.1016/j.jse.2015.11.016

32. Gierer $P$ et al (2010) Microcirculatory sequelae of the rotator cuff after antegrade nailing in proximal humerus fracture. Arch Orthop Trauma Surg 130:687-691

33. Sosef N, Leerdam Rvan, OttP, Meylaerts S, Rhemrev $S$ (2010) Minimal invasive fixation of proximal humeral fractures with an intramedullary nail: good results in elderly patients. Arch Orthop Trauma Surg 130:605-611

34. Han RJ, Sing DC, Feeley BT, Ma CB, Zhang AL (2016) Proximal humerus fragility fractures: recent trends in nonoperative and operative treatment in the medicare population. J Shoulder Elbow Surg 25:256-261

35. Wang J et al (2016) Meta-analysis suggests that reverse shoulder arthroplasty in proximal humerus fractures is a better option than hemiarthroplasty in the elderly. Int Orthop 40:531-539

36. Rabi S, Evaniew N, Sprague SA, Bhandari M, Slobogean GP (2015) Operative vs non-operative management of displaced proximal humeral fractures in the elderly: A systematic review and meta-analysis of randomized controlled trials. World J Orthop 6:838-846

37. Capriccioso CE, Zuckerman JD, EgolKA (2016) Initial varus displacement of proximal humerus fractures results in similar function but higher complication rates. Injury. doi:10.1016/j.injury.2016.01.021

38. Tamimi I et al (2015) Displaced proximal humeral fractures: when is surgery necessary? Injury 46:1921-1929 